\title{
A Case of Human Hepatic Alveolar Echinococcosis Accompanied by Lung and Brain Metastases
}

\author{
Chuanchuan Liu ${ }^{1,2,3} \mathbb{C}$, Haining Fan ${ }^{2,3} \mathbb{0}$, Ri-li Ge $e^{1,2,3, *}$ (i) \\ ${ }^{1}$ Research Center for High Altitude Medicine, Qinghai University, Xining, Qinghai 810001, China; ${ }^{2}$ Qinghai University Affiliated Hospital, Xining, \\ Qinghai 810001, China; ${ }^{3}$ Qinghai Key Laboratory for Echinococcosis, Xining, Qinghai 810001, China
}

\begin{abstract}
Alveolar echinococcosis (AE) is considered as a fatal zoonosis caused by the larvae of Echinococcus multilocularis. The lungs and brain are the most common metastatic organs. We report a human case of hepatic alveolar echinococcosis accompanied by lung and brain metastasis. In particular, the patient had a history of tuberculosis and the lung lesions were easily misdiagnosed as lung abscesses. The lesions of liver and lung underwent radical resection and confirmed as alveolar echinococcosis by pathological examination. The patient had no surgical complications after operation and was discharged after symptomatic treatment. Unfortunately, the patient later developed multiple intracerebral AE metastases. We required the patient to take albendazole orally for life and follow up.
\end{abstract}

Key words: Echinococcus multilocularis, alveolar echinococcosis, human, misdiagnosis

\section{INTRODUCTION}

Alveolar echinococcosis (AE) is a zoonotic disease that primarily affects the liver but has the potential for local invasion and metastasis to brain, lung and bones. AE is distributed worldwide, mainly in the northern hemisphere [1,2]. Even though $\mathrm{AE}$ is a benign disease caused by parasites in histopathology, it shows the growth characteristics of malignant tumors [2]. Almost all AE originates in the liver and can spread to distant organs such as the lungs, brain, and kidneys through blood circulation $[3,4]$. Human are not suitable intermediate hosts for E. multilocularis, and the presence of the protoscolices is rarely observed in the lesions. In the present case, we report a case of primary hepatic AE with lung and brain metastases. The patient had a history of Mycobacterium tuberculosis infection, and the imaging features of lung lesions showed pulmonary abscess. As a result, we were misdiagnosed. The patient underwent radical resection of liver and lung lesion and was treated with albendazole regularly after operation. The patient had multiple AE metastases in the brain about one year after the lung lesion surgery. Eventually, the patient lost a chance to

- Received 28 October 2020, revised 5 February 2021, accepted 9 February 2021.

*Corresponding author (geriligao@hotmail.com)

(c) 2021, Korean Society for Parasitology and Tropical Medicine

This is an Open Access article distributed under the terms of the Creative Commons Attribution Non-Commercial License (https://creativecommons.org/licenses/by-nc/4.0) which permits unrestricted non-commercial use, distribution, and reproduction in any

medium, provided the original work is properly cited. treat due to multiple intracranial AE lesions.

\section{CASE RECORD}

Written informed consent was obtained from patient and the article was approved by the Ethics Committee of the Affiliated Hospital of Qinghai University (AF-RHEC-0018-01).

On January 3, 2017, a 34-year-old Tibetan Chinese man came to the Department of Neurosurgery at the Qinghai University Affiliated Hospital for treatment with severe upper abdominal pain. The patient lived in pastoral areas of Qinghai Province for a long time and had been in good health before. Physical examination touched a mass in the upper abdomen, which was $18.0 \times 16.0 \mathrm{~cm}$ in size, accompanied by tenderness. Ultrasound Color Doppler of the upper abdomen presented a $11.6 \times 11.2 \mathrm{~cm}$ mixed mass in the right posterior lobe of the liver (Fig. 1A). The dynamic 3-phase Computed Tomography (CT) examination of the liver demonstrated a round lesions (about $9.6 \times 8.9 \mathrm{~cm}$ ) in the liver $57-6$ segment, with a thick wall, and a CT value of about 16 HU. Calcification could be observed in the lesion, and no enhancement was seen in the lesion after enhancement (Fig. 1B). Chest X-ray and laboratory findings were all within normal limit. Additionally, hydatid serological IgG ELISA remained negative. Stool examination showed no parasites or eggs. Based on these findings, the patient was diagnosed as hepatic $\mathrm{AE}$. The patient underwent right hepatectomy and cholecystectomy on September 6, 2017. 

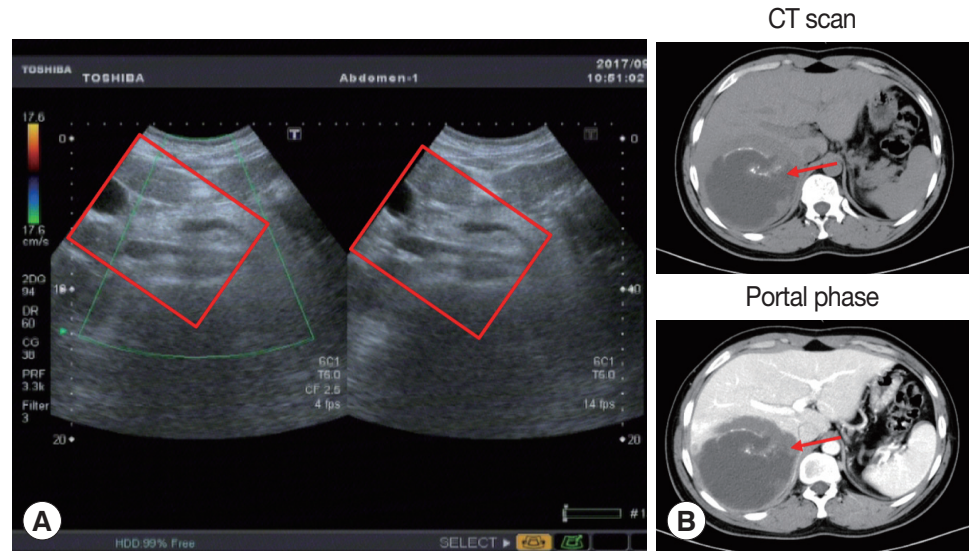

Portal phase
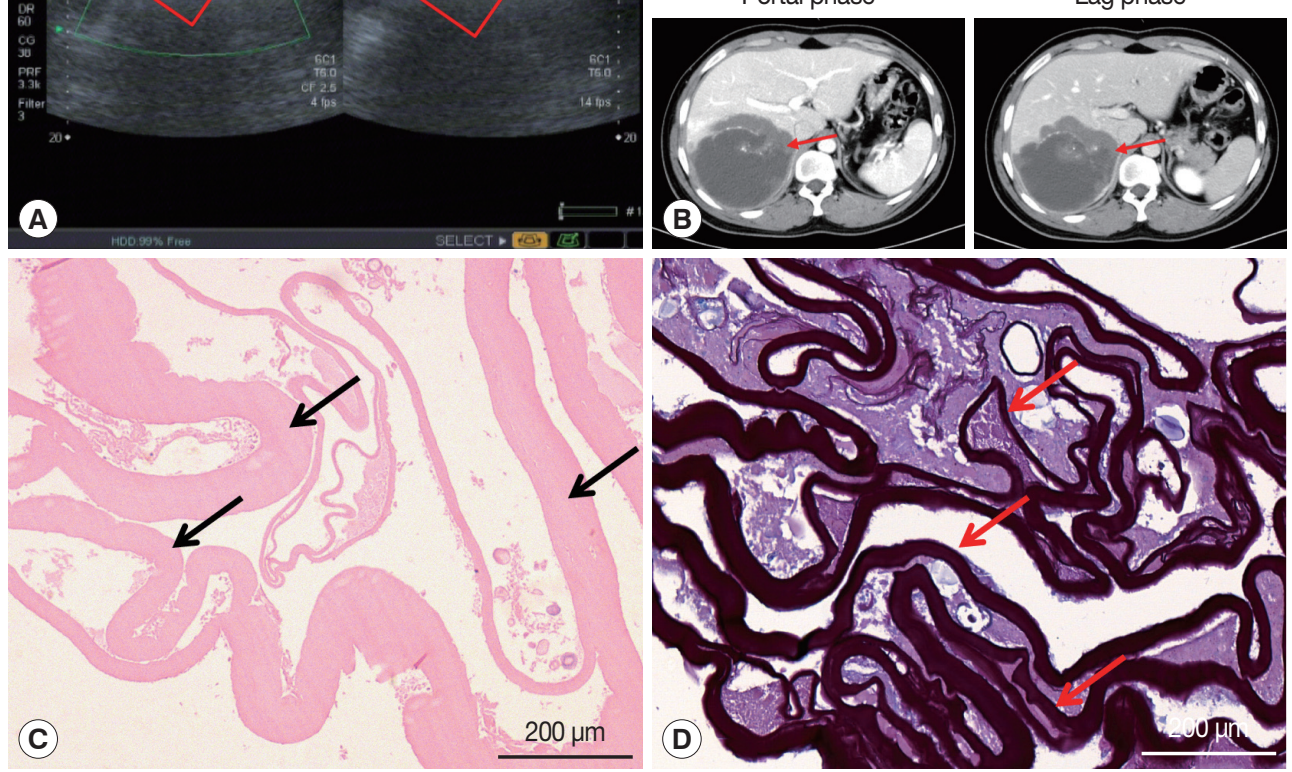

Fig. 1. Imaging and pathological findings of liver lesions. (A) Color Doppler ultrasonography of the liver. A hybrid mass $11.6 \times 11.2 \mathrm{~cm}$ was observed in the right posterior lobe of the liver (red box). (B) Abdominal dynamic phase III computed tomography (red arrow). Round low-density shadows were seen in the right lobe of the liver, and no enhancement was observed after contrast-enhanced scan. Highdensity nidi were scattered in the lesion. The maximum cross-section of the lesion is about $9.6 \times 8.9 \mathrm{~cm}$. (C) The hematoxylin and eosin stain of paraffin sections displayed the laminated layer (black arrows). (D) Periodic acid-Schiff (PAS) stain presented a strongly PAS-positive basophilic laminated layer (red arrows).

During the operation, there existed obvious adhesion between the lesion and the lateral abdominal wall, which invaded the diaphragm and lateral peritoneum. Postoperative pathological examination confirmed AE disease. The histopathological examination showed obvious laminated layer (Fig. 1C), intensely colored by the periodic acid-Schiff stain without a germinal layer (Fig. 1D). These morphological findings verified AE. The patient fully recovered after surgery. The patient was treated with albendazole (400 mg twice a day) orally for 2 year.

On May 13, 2019, the patient came to our hospital due to cough and chest pain for 15 days. The patient had hemoptysis once with obvious weight loss. Tissue components were found in the patient's sputum. Oxygen saturation was about $60 \%$. Chest CT showed an encapsulated lesion (about $8.4 \times 6.9 \mathrm{~cm}$ ) in the lower lobe of the right lung, considering the possibility of abscess formation (Fig. 2A). CT scan of brain showed no abnormalities. The patient's hydatid serological IgG test and $\gamma$
- interferon release test remained positive. In addition, any binding bacteria were not in sputum. Laboratory examination proved that the C-reactive protein was $20.50 \mathrm{mg} / \mathrm{L}$ (normal value $0-6)$, and the erythrocyte sedimentation rate was 30 $\mathrm{mm} / \mathrm{hr}$ (normal value $0-15$ ). The patient was considered to have a lung abscess caused by previous tuberculosis infection. The patient underwent radical resection of the lower lobe of the right lung on May 21, 2019. During the operation, there existed obvious adhesion in the right thoracic cavity, and an $8.0 \times 8.0 \mathrm{~cm}$ abscess cavity was seen in the basal segment of the right lower lobe, which was filled with purulent fluid. The lower lobe and the diaphragm were adhered with no obvious boundary. The right lower pulmonary ligament, pulmonary artery, pulmonary vein and bronchus were ligated and cut off. The diaphragmatic adhesions were carefully separated and the lesions were completely removed. The pathological sections revealed the laminated membranes and protoscoleces (Fig. 

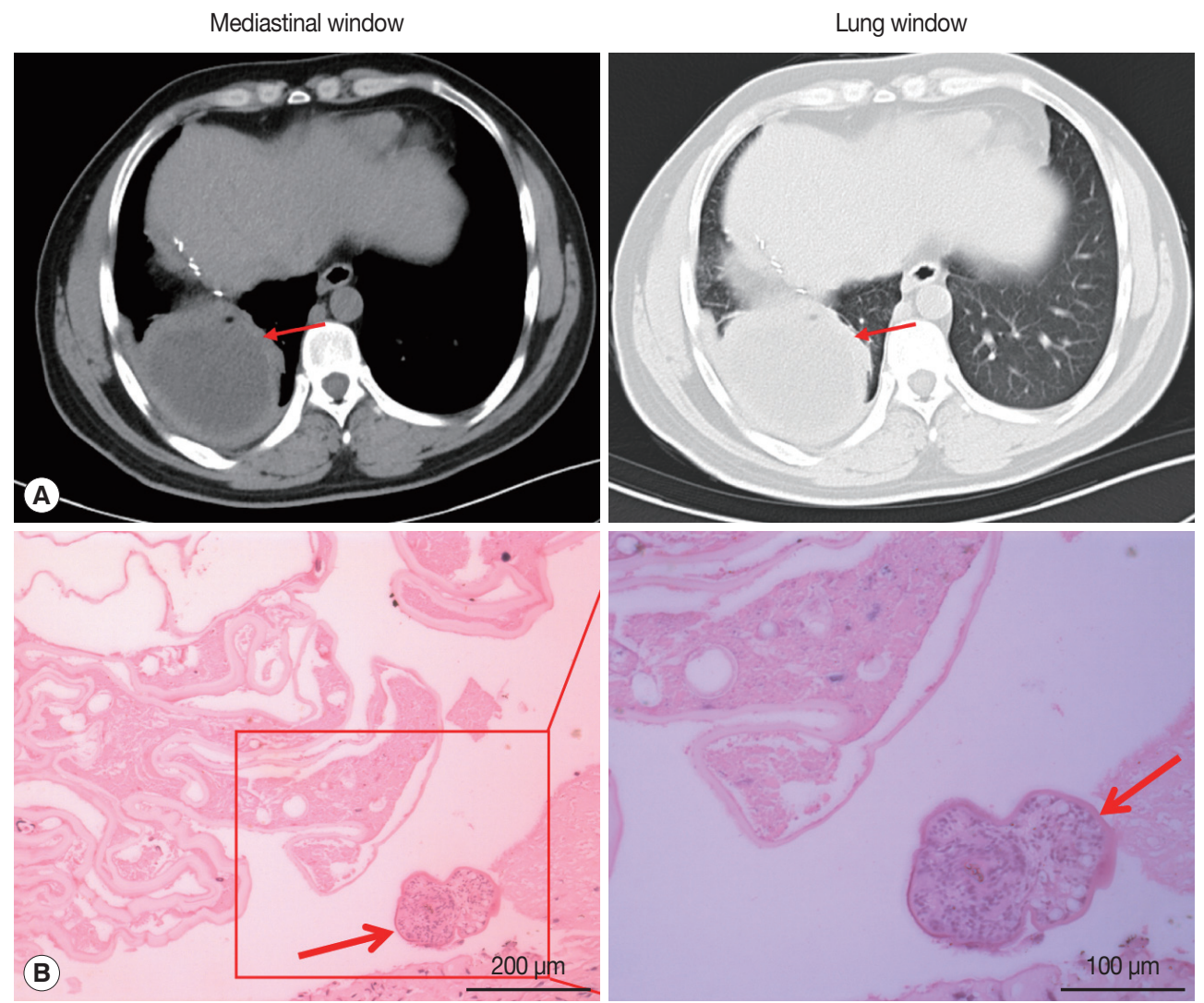

Fig. 2. Imaging and pathological findings of pulmonary lesions. (A) Thorax computed tomography scan revealed an $8.4 \times 6.9 \mathrm{~cm}$ encapsulated lesion in the right lung (red arrow). (B) Pathological finding of lung excision lesion. A protoscolex (red arrows) was observed in the lesion.

2B). The patient's pulmonary complications disappeared and pulmonary function completely recovered after surgery. we recommended that the patient to take albendazole orally (400 mg twice a day) for at least 2 years.

On March 30, 2020, the patient came to the neurosurgery department of our hospital again due to unprovoked dizziness and headache for 2 weeks. The patient suspected that he had an upper respiratory tract infection and took oral medication to treat it by himself (the specific medication was unknown). In the past 1 week, accompanied by nausea and vomiting, the patient had frequent headaches and blurred vision in his right eye. Physical examination of right limb muscle strength gave grade IV, and unclear vision in right eye. Brain CT images showed multiple round lesions in the brain parenchyma (Supplementary Fig. S1). According to MRI examination, there were multiple short $\mathrm{T} 2$ and other $\mathrm{T} 1$ signal lesions in the brain parenchyma with ring enhancement in the brain parenchyma, and obvious edema around the lesions (Fig. 3). The patient's vital signs and laboratory tests were in the normal range. Com- bined with the patient's medical history, the patient was clearly diagnosed as cerebral AE metastasized from the primary lesion. The multiple AE lesions in the brain were in the regions difficult to remove by operation. In addition, the patient had a grand mal seizure during hospitalization. When informing the current condition to his family members, they gave up further treatment and discharged the patient. At discharge, we recommended that the patient to take albendazole $400 \mathrm{mg}$ twice a day for life

\section{DISCUSSION}

Alveolar echinococcosis is a serious and fatal helminth disease with an increasing incidence in endemic areas [5]. E. multilocularis adults mainly parasitize in the intestines of foxes, wolves and dogs, and the eggs excreted can contaminate food and water and infect human beings. The incubation period of $\mathrm{AE}$ infection is about 5-15 years, and there is no early clinical symptoms. The disease is usually diagnosed in the late stage, 

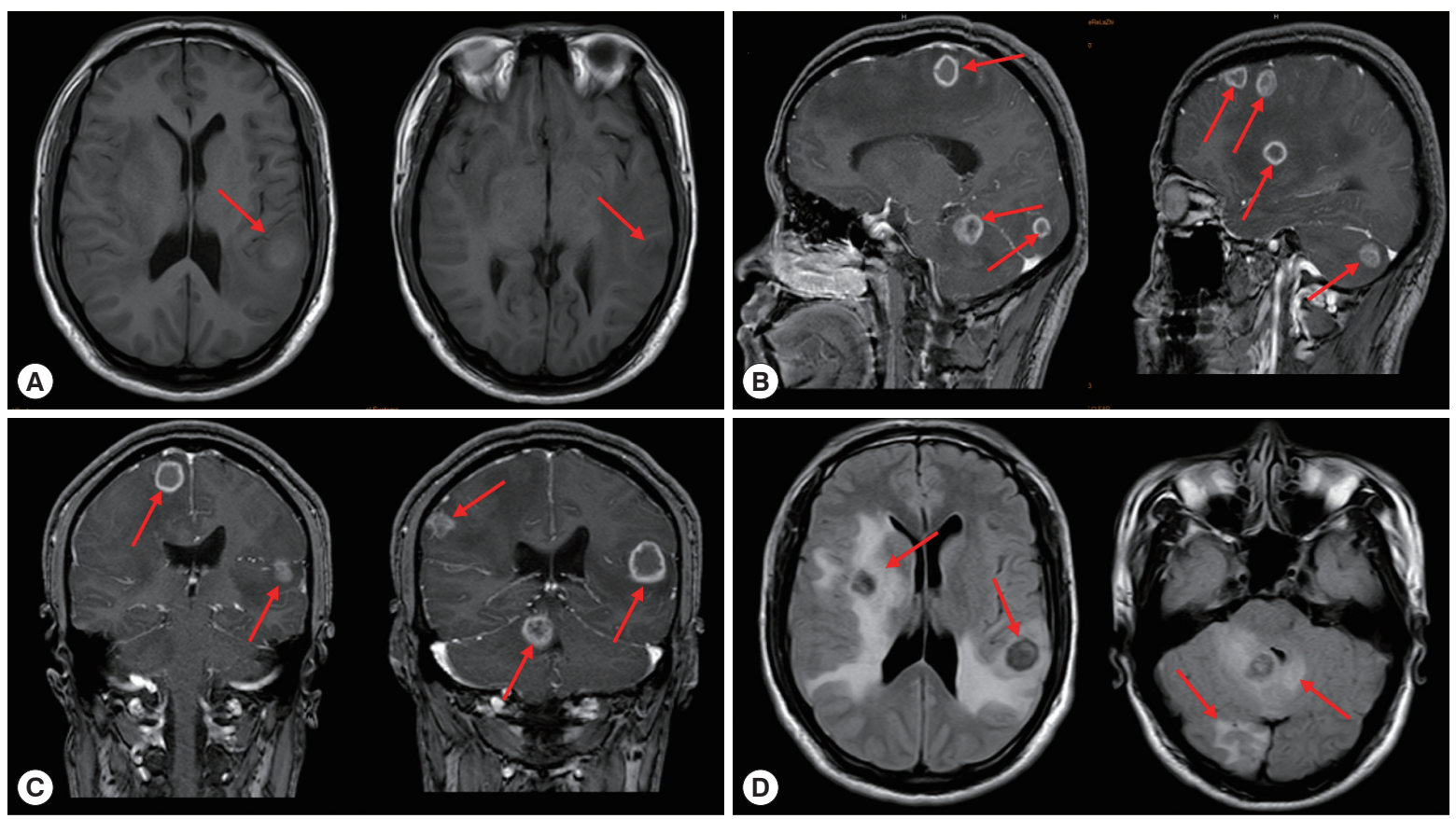

Fig. 3. Brain magnetic resonance imaging. (A) Axial view T1-weighted image showed the irregular round lesions in the brain with an extensive perifocal edema (red arrows). Sagittal view T2-weighted image (B), coronal T2-weighted image (C) and axial view T2-weighted image (D) with contrast enhancement showing the multiple lesions with a perifocal edema (red arrows).

and only a small number of patients can undergo the complete surgical resection [6]. The diagnosis of $\mathrm{AE}$ needs to be combined with epidemiological history, clinical manifestations, imaging examination and serological examination [7].

According to its life cycle, human $\mathrm{AE}$ is almost $100 \%$ primary in liver, and can be transferred to lung, brain, heart, peritoneum and other parts through blood and lymphatic system in later stage [8-11]. Central nervous system involvement is considered to be a marker of the terminal stage of $\mathrm{AE}$ [12]. Additionally, our patient also shows obvious lung and brain metastases. Ultrasound Color Doppler has a high sensitivity in the diagnosis of hepatic vesicular hydatid disease and is often used as the first choice for preliminary diagnosis $[13,14]$. CT and MRI have important clinical value in diagnosis, postoperative follow-up, and judgment of calcification of AE [15,16]. The lesions of AE show no obvious boundary on ultrasound, CT, and MRI [17]. Our patient showed evident calcification on CT in the liver, with unclear peripheral boundaries and uninvaded portal veins. During the operation, it could be observed that the portal vein was not invaded, while the diaphragm was visibly violated. The patient underwent radical resection and longterm oral albendazole treatment. Despite these treatments, the patient was diagnosed with lung AE 2 years later.
Hepatic AE metastasis to the lung is relatively high, observed in $7-20 \%$ of the cases [18]. The clinical symptoms of lung $\mathrm{AE}$ are mostly hemoptysis, chest pain, cough with sputum, and exertional dyspnea. Our patient also has these symptoms. However, CT imaging of the patient's lung lesions showed signs of lung abscess, with a thicker cyst wall, similar to the imaging findings of cystic echinococcosis [19]. The patient's serum hydatid IgG and $\gamma$-interferon release test were positive. Imaging examination found no new lesions in the patient's brain and liver. As a result, the patient's AE was misdiagnosed as a lung abscess caused by old tuberculosis until it was confirmed by pathological examination. During the operation, the lung lesions were observed to adhere to the surrounding lung tissues, and there were tangible substances in the patient's sputum, which may be caused by the lesions invading the bronchus. Although serological test has low sensitivity and specificity in the diagnosis of hydatid disease, it is useful in the diagnosis of hydatid disease and postoperative follow-up [20].

The clinical symptoms of brain AE depends on the degree of damage to central nervous system, including common symptoms of increased intracranial pressure, epilepsy, neurological disorders (such as blurred vision and paralysis), and cranial nerve palsy [21]. The CT and MRI of cerebral AE are mostly 
solid or multilocular cystic masses with clear boundaries, accompanied by calcification and peripheral edema [4]. Our patient also presented obvious nervous system involvement. CT and MRI of the patient showed multiple solid intracranial lesions with edema. The incubation period of $\mathrm{AE}$ infection is about 5-15 years, during which there are no obvious clinical symptoms. The patient's brain lesions may have been metastasized due to liver lesion or rupture of a lung abscess. The patient currently depend on taking albendazole oral treatment for a long time. Albendazole can penetrate the blood-brain barrier more than mebendazole and is also the first choice of chemotherapy drugs for brain $\mathrm{AE}$ [22].

$\mathrm{AE}$ is a potentially fatal parasitic disease which is often misdiagnosed. The diagnosis depends on the comprehensive judgment by several diagnostic methods including imaging, serology, epidemiology, and histopathology. For metastatic AE, especially when the imaging features of the metastatic lesions are not typical, the previous history of echinococcosis provides great reference value for its diagnosis. Unfortunately, our patient has multiple brain AE metastases after 3 years of diagnosis. Currently, we only hope that relying on chemical drug treatment can enhance the quality of life of the patient.

\section{ACKNOWLEDGMENT}

Project supports were provided by the Qinghai Provincial Natural Science Foundation (2020-ZJ-956Q) and the National key R \& D projects (2017YFC0909900).

\section{CONFLICT OF INTEREST}

We declare no conflict of interest related to this study.

\section{REFERENCES}

1. Sade R, Kantarci M, Ogul H, Gundogdu B, Aydınlı B. Differentiation between hepatic alveolar echinococcosis and primary hepatic malignancy with diffusion-weighted magnetic resonance imaging. Diagn Interv Imaging 2018; 99: 169-177. https://doi.org/10.1016/ j.diii.2017.09.007

2. Liu W, Delabrousse É, Blagosklonov O, Wang J, Zeng H, Jiang Y, Wang J, Qin Y, Vuitton DA, Wen H. Innovation in hepatic alveolar echinococcosis imaging: best use of old tools, and necessary evaluation of new ones. Parasite 2014; 21: 74. https://doi.org/10.1051/ parasite/2014072

3. Spahn S, Helmchen B, Zingg U. Alveolar echinococcosis of the right adrenal gland: a case report and review of the literature. J Med
Case Rep 2016; 10: 325. https://doi.org/10.1186/s13256-016-1115-0

4. Atalan G, Sivrioglu AK, Sönmez G, Celik M, Simsek B. A case of alveolar echinococcosis presenting as cerebral and spinal intradural metastases. Eurasian J Med 2016; 48: 149-152. https://doi.org/10.5152/ eurasianjmed.2015.15003

5. Salm LA, Lachenmayer A, Perrodin SF, Candinas D, Beldi G. Surgical treatment strategies for hepatic alveolar echinococcosis. Food Waterborne Parasitol 2019; 15: e00050. https://doi.org/10.1016/ j.fawpar.2019.e00050

6. Schmidberger J, Steinbach J, Schlingeloff P, Kratzer W, Grüner B. Surgery versus conservative drug therapy in alveolar echinococcosis patients in Germany-A health-related quality of life comparison. Food Waterborne Parasitol 2019; 16: e00057. https://doi.org/10.1016/ j.fawpar.2019.e00057

7. Kayacan SM, Vatansever S, Temiz S, Uslu B, Kayacan D, Akkaya V, Erk O, Saka B, Karadag A, Turkmen K, Fatih Y, Kerim G. Alveolar echinococcosis localized in the liver, lung and brain. Chin Med J (Engl) 2008; 121: 90-92.

8. Kantarci M, Bayraktutan U, Pirimoglu B, Ogul H, Oral A, Eren S, Gundogdu B. Multisystem involvement of alveolar echinococcosis in a child. J Infect Dev Ctries 2014; 8: 1494-1497. https://doi.org/10.3855/ jidc. 4214

9. Schmidberger J, Kratzer W, Stark K, Grüner B. Alveolar echinococcosis in Germany, 1992-2016. An update based on the newly established national AE database. Infection 2018; 46: 197-206. https:// doi.org/10.1007/s15010-017-1094-0

10. Wang $\mathrm{H}$, Lu C, Liu X, Zhang W. Metastatic and prognostic factors in patients with alveolar echinococcosis. Int J Clin Exp Pathol 2015; 8: 11192-11198.

11. McManus DP, Gray DJ, Zhang W, Yang Y. Diagnosis, treatment, and management of echinococcosis. BMJ 2012; 344: e3866. https://doi. org/10.1136/bmj.e3866

12. Bresson-Hadni S, Delabrousse E, Blagosklonov O, Bartholomot B, Koch S, Miguet JP, Mantion GA, Vuitton DA. Imaging aspects and non-surgical interventional treatment in human alveolar echinococcosis. Parasitol Int 2006; 55 (suppl): 267-272. https://doi.org/10.1016/ j.parint.2005.11.053

13. Sulima M, Nahorski W, Gorycki T, Wołyniec W, Wąż P, Felczak-Korzybska I, Szostakowska B, Sikorska K. Ultrasound images in hepatic alveolar echinococcosis and clinical stage of the disease. Adv Med Sci 201; 64: 324-330. https://doi.org/10.1016/j.advms.2019.04.002

14. Kratzer W, Reuter S, Hirschbuehl K, Ehrhardt AR, Mason RA, Haenle MM, Kern P, Gabelmann A. Comparison of contrast-enhanced power Doppler ultrasound (Levovist) and computed tomography in alveolar echinococcosis. Abdom Imaging 2005; 30: 286-290. https://doi.org/10.1007/s00261-004-0263-7

15. Aoki T, Hagiwara M, Yabuki $H$, Ito A. Unique MRI findings for differentiation of an early stage of hepatic alveolar echinococcosis. BMJ Case Rep 2015; 2015: bcr2014208123. https://doi.org/10.1136/bcr2014-208123

16. Graeter T, Kratzer W, Oeztuerk S, Haenle MM, Mason RA, Hillenbrand A, Kull T, Barth TF, Kern P, Gruener B. Proposal of a computed tomography classification for hepatic alveolar echinococcosis. 
World J Gastroenterol 201; 22: 3621-3631. https://doi.org/10.3748/ wjg.v22.i13.3621

17. Jiang C. Two cases of liver alveolar echinococcosis associated with simultaneous lung and brain metastases. Chin Med J (Engl) 2002; 115: 1898-1901.

18. Kantarci M, Bayraktutan U, Karabulut N, Aydinli B, Ogul H, Yuce I, Calik M, Eren S, Atamanalp SS, Oto A. Alveolar echinococcosis: spectrum of findings at cross-sectional imaging. Radiographics 2012; 32: 2053-2070. https://doi.org/10.1148/rg.327125708

19. Armoon A, Mehrian P, Soleimantabar H, Torabi S, Ghahderijani BH. Computer tomography (CT) characteristics of pulmonary cystic echinococcosis. Med Arch 2019; 73: 338-343. https://doi.org/10.5455/ medarh.2019.73.338-343
20. Belahcen M, Khattala K, Elmadi A, Bouabdellah Y. Cerebral hydatid cyst in children: report of 5 cases. Pan Afr Med J 2014; 17: 149 (in French). https://doi.org/10.11604/pamj.2014.17.149.3419

21. Algros MP, Majo F, Bresson-Hadni S, Koch S, Godard J, Cattin F, Delbosc B, Kantelip B. Intracerebral alveolar echinococcosis. Infection 2003; 31: 63-65. https://doi.org/10.1007/s15010-002-2178-y

22. Kvascevicius R, Lapteva O, Awar OA, Audronyte E, Neverauskiene L, Kvasceviciene E, Sokolovas V, Strupas K, Marcinkute A, Deplazes $\mathrm{P}$, Mehmet Tt. Fatal liver and lung alveolar echinococcosis with newly developed neurologic symptoms due to the brain involvement. Surg J (NY) 2017; 2: e83-83e88. https://doi.org/10.1055/ s-0036-1592122 